\title{
An interacting system in population genetics, II
}

\author{
By \\ Tokuzo SHIGA \\ (Communicated by Prof. S. Watanabe, Aug. 14, 1979)
}

\section{Introduction}

In the previous paper [10] we studied an interacting system in population genetics, which is called a continuous time stepping stone model. Let us review our model. Let $S$ be a countable set. Each element $i$ of $S$ is called a colony. Assuming that there are two alleles $A$ and $B$ at each colony, we denote by $x_{i}$ $\left(1-x_{i}\right)$ the gene frequency of the $A$-allele (resp. the $B$-allele) for the colony $i \in S$. We consider a time evolution of gene frequencies, which is caused by migration among colonies and random sampling drift.

Let $X=\left[\begin{array}{ll}0 & 1\end{array}\right]^{S}$ be the space of systems of gene frequencies, which is equipped with the product topology. Let $C(X)$ be the Banach space of all continuous functions equipped with the supremum norm and $C_{0}^{2}(X)$ be the set of all $C^{2}$-functions depending only on finite number of coordinates of $X$.

Let us consider the following infinite dimensional differential operator $A$,

$$
A f(x)=\sum_{i \in S} \frac{1}{4 N} x_{i}\left(1-x_{i}\right) \frac{\partial^{2} f}{\partial x_{i}^{2}}+\sum_{i \in S}\left(\sum_{j \in S} q_{i j} x_{j}\right) \frac{\partial f}{\partial x_{i}},
$$

where $N>0$ and $q_{i j}(i, j \in S)$ are constants such that $q_{i j} \geqq 0$ for $i \neq j$ and $\sum_{j \in S} q_{i j}=0$ for each $i \in S$.

Let $\left\{T_{t}\right\}$ be a strongly continuous semi-group on $C(X)$ such that

$$
T_{t} 1=1 \text { and } T_{t} f \geqq 0 \quad \text { for every } f \in C(X) \text { satisfying } f \geqq 0 \text {, }
$$

and

$$
T_{t} f-f=\int_{0}^{t} T_{s} A f d s \quad \text { for every } f \in C_{0}^{2}(X) .
$$

Such a semi-group $\left\{T_{\imath}\right\}$ is uniquely determined under the following assumption,

$$
\sup _{i \in S}\left|q_{i i}\right|<+\infty . \quad \text { (cf. [10], [11]). }
$$

Here $N$ means the effective population size of each colony and $q_{i j}(i \neq j)$ means the migration rate from $j \in S$ to $i \in S$.

Then $\left\{T_{t}\right\}$ defines a diffusion process on $X$, which we call a continuous time stepping stone model without mutation and selection.

Discrete time stepping stone models were first proposed by M. Kimura and 
they have been investigated by many biologists. cf. [1], [3], [7], [8], [9], etc.. However the problems of stationary states and ergodic behaviors seems to be difficult for discrete time models with infinite colonies.

On the other hand the stepping stone models can be regarded as interesting examples of infinitely interacting systems, which have been studied extensively for the last decade. (see e. g. [5]).

In particular dual processes, which are often used in the theory of infinitely interacting systems, are powerful tools also in our model.

In the previous paper [10] we introduced the following classification by the migration rates $Q=\left\{q_{i j}\right\}$. Let $P_{t}=\exp t Q$ for any $t \geqq 0$, and $P_{t} \otimes P_{t}$ is defined by

$$
\begin{array}{r}
P_{t} \otimes P_{t}(\vec{i}, \vec{j})=P_{t}\left(i_{1}, j_{1}\right) P_{t}\left(i_{2}, j_{2}\right) \quad \text { for each } \vec{i}=\left(i_{1}, i_{2}\right) \in S \times S \\
\text { and } \vec{j}=\left(j_{1}, j_{2}\right) \in S \times S .
\end{array}
$$

We denote by $\left(X_{t}=\left(X_{t}^{1}, X_{t}^{2}\right), P_{\vec{i}}^{(2)}\right)_{\vec{i} \in S \times S}$ the continuous time Markov chain on $S \times S$, associated with $P_{t} \otimes P_{t}$. We assume that $Q=\left\{q_{i j}\right\}$ is irreducible.

Case I. $\quad P_{\vec{i}}^{(2)}\left[\int_{0}^{\infty} I_{\Delta_{2}}\left(X_{t}\right) d t=+\infty\right]=1 \quad$ for all $\vec{i} \in S \times S$,

Case II. $\quad P_{\vec{i}}^{(2)}\left[\int_{0}^{\infty} I_{\Delta_{2}}\left(X_{t}\right) d t=+\infty\right]=0 \quad$ for all $\vec{i} \in S \times S$,

Case III. $0<\underset{\vec{i}}{P_{\vec{i}}^{(2)}}\left[\int_{0}^{\infty} I_{\Delta_{2}}\left(X_{t}\right) d t=+\infty\right]<1 \quad$ for all $\vec{i} \in S \times S$,

where $\Delta_{2}=\left\{\left(i_{1}, i_{2}\right) \in S \times S \mid i_{1}=i_{2}\right\}$ and $I_{\Delta_{2}}$ denotes the indicator function of $\Delta_{2}$. Since $Q$ is irreducible, Case $I$, Case $I I$, and Case $I I I$ exhaust all possibilities.

In [10], we solved the problems of stationary states and ergodic behaviors in Case I and Case II. In this paper we shall investigate the general case, including Case III. The dual process of our model is similar to that in a voter model of Holley \& Liggett [2] and also the stationary states of our model can be described in the same fashion as the voter model.

Let us introduce the space of $P_{t}$-harmonic functions $\mathscr{H}$ and a sub-class $\mathscr{H}^{*}$ of $\mathscr{T}$.

$$
\mathscr{H}=\left\{h ; \text { defined on } S, 0 \leqq h \leqq 1 \text {, and } P_{t} h=h \text { for all } t>0\right\},
$$

and

$$
\begin{aligned}
\mathscr{H}^{*}= & \left\{h \in \mathcal{H} ; \lim _{t \rightarrow \infty} h\left(X_{t}^{1}\right)=\lim _{t \rightarrow \infty} h\left(X_{t}^{2}\right)=0 \text { or } 1,\left(P_{\vec{i}}^{(2)}\right) \text {-almost surely on } \Omega^{(1)},\right. \\
& \text { for each } i \in S \times S\}, \text { where } \Omega^{(1)}=\left[\int_{0}^{\infty} I_{\Delta_{2}}\left(X_{t}\right) d t=+\infty\right] .
\end{aligned}
$$

If we endow $\mathscr{H}$ with the topology of point-wise convergence, $\mathscr{H}$ is a compact convex set. So, let us denote by $\mathscr{H}_{e x}$ the set of all extremal elements of $\mathscr{T}$. Then, it holds generally that $\mathscr{H}_{e x} \subseteq \mathscr{H}^{*} \subseteq \mathscr{H}$. In particular $\mathscr{H}^{*}=\mathscr{H}$ holds in Case $I I$, because of $P_{\vec{i}}^{(2)}\left[\Omega^{(1)}\right]=0$ for each $\vec{i} \in S \times S$.

Let $\mathscr{P}(X)$ be the set of all probability measures on $X$ equipped with the topology of weak convergence. Let us denote by $\left\{T_{t}^{*}\right\}$ the adjoint semi-group on 
$\mathscr{P}(X)$ induced by $\left\{T_{t}\right\}$ and $\mathcal{S}$ denotes the set of all stationary states, i. e. $\mathcal{S}=$ $\left\{\mu \in \mathscr{P}(X) ; T_{t}^{*} \mu=\mu\right.$ for all $\left.t \geqq 0\right\}$. Since $\mathcal{S}$ is a compact convex set, we shall investigate the structure of $\mathcal{S}_{e x}$, the set of all extremal elements of $\mathcal{S}$.

Then the following theorems hold.

Theorem 1.1.

(1) For each $h \in \mathscr{H}$, there exists a $\nu_{h} \in \mathscr{P}(X)$ such that $\lim _{t \rightarrow \infty} T_{t}^{*} \delta_{h}=\nu_{h}$, where $\delta_{h}$ stands for the point mass at $h$,

(2) $\int_{X} x_{i} \nu_{h}(d x)=h(i) \quad$ for each $h \in \mathscr{H}$ and $i \in S$,

and

(3) $\mathcal{S}_{e x}=\left\{\nu_{h} ; h \in \mathscr{H}^{*}\right\}$.

Theorem 1.2. Let $\mu \in \mathscr{P}(X)$ and $h \in \mathscr{H}^{*}$. Then, $\lim T_{l}^{*} \mu=\nu_{h}$ if and only if

$$
\lim _{t \rightarrow \infty} \sum_{j \in S} P_{t}(i, j) \int_{X} x_{j} \mu(d x)=h(i) \quad \text { for each } i \in S \text {, }
$$

and

$$
\lim _{t \rightarrow \infty} \sum_{j \in S} \sum_{k \in S} P_{t}(i, j) P_{t}(i, k) \int_{X} x_{j} x_{k} \mu(d x)=h(i)^{2} \quad \text { for each } i \in S .
$$

\section{Remark.}

(i) In Case I it holds that $\mathscr{H}^{*}=\mathscr{M}_{e x}=\{0,1\}$.

(ii) In Case II it holds that $\mathscr{H}^{*}=\mathscr{H} \supseteqq\{c ; 0 \leqq c \leqq 1\}$.

(iii) In Case III it holds that $\mathscr{H}_{e x} \subseteq \mathscr{H}^{*} \varsubsetneqq \mathscr{H}$.

In fact, we have examples of Case III, for which $\mathscr{H}_{e x} \subsetneq \mathscr{A}^{*}$ holds.

In $\S 2$ we shall introduce a dual process of our model and discuss some properties on it. $\$ 3$ will be devoted to the proof of the above theorems, and in the final section some examples will be given.

Acknowledgement. The author would like to thank to T.M. Liggett who kindly showed him the proof of the final theorem in [2].

\section{Dual processes}

Let $I$ be the set of all non-negative integer-valued functions $\alpha$ defined on $S$, satisfying $|\alpha|=\sum_{i \in S} \alpha_{i}<+\infty . \quad \alpha$ is denoted by $\varepsilon^{i}$ if $\alpha_{i}=1$ and $\alpha_{j}=0$ for $j \neq i$. For $\alpha \in I$ and $\beta \in I, \alpha+\beta$ and $\alpha-\beta$ are defined component-wise.

Let us introduce two kind of continuous time Markov chains $\left(\alpha_{t}, \boldsymbol{P}_{\alpha}\right)_{\alpha \in I}$ and $\left(\alpha_{t}, \tilde{\boldsymbol{P}}_{\alpha}\right)_{\alpha \in I}$, which are generated by the following infinitesimal matrices $\left\{R_{\alpha, \beta}\right\}$ and $\left\{\widetilde{R}_{\alpha, \beta}\right\}$ on $I \times I$, respectively. 


$$
\begin{gathered}
\text { Tokuzo Shiga } \\
R_{\alpha, \beta}= \begin{cases}\alpha_{i} q_{i j} \quad \text { if } \beta=\alpha-\varepsilon^{i}+\varepsilon^{j} \in I \quad(i \neq j), \\
\frac{1}{4 N} \alpha_{i}\left(\alpha_{i}-1\right) \quad \text { if } \beta=\alpha-\varepsilon^{i} \in I, \\
\sum_{i \in S} \alpha_{i} q_{i i}-\frac{1}{4 N} \sum_{i \in S} \alpha_{i}\left(\alpha_{i}-1\right) \quad \text { if } \beta=\alpha, \\
0 \quad \text { otherwise. }\end{cases} \\
\tilde{R}_{\alpha, \beta}= \begin{cases}\alpha_{i} q_{i j} \quad \text { if } \beta=\alpha-\varepsilon^{i}+\varepsilon^{j} \in I \quad(i \neq j), \\
\sum_{i \in S} \alpha_{i} q_{i i} \quad \text { if } \beta=\alpha, \\
0 \quad \text { otherwise. }\end{cases}
\end{gathered}
$$

Here we note that $\left(\alpha_{t}, \tilde{\boldsymbol{P}}_{\alpha}\right)_{\alpha \in I}$ can be regarded as an independent system of $P_{t^{-}}$ Markov chains and $\left(\alpha_{t}, \boldsymbol{P}_{\alpha}\right)_{\alpha \in I}$ has the same probability law as $\left(\alpha_{t}, \tilde{\boldsymbol{P}}_{\alpha}\right)_{a \in I}$ up to the hitting time for $\Delta$, where $\Delta=\left\{\alpha \in I ; \alpha_{i} \geqq 2\right.$ for some $\left.i \in S\right\}$.

Also, $\left(\alpha_{t}, \boldsymbol{P}_{\alpha}\right)_{\alpha \in I}$ is a dual process of $\left\{T_{t}\right\}$ in the following sense. Let us define a family of functions $\left\{f_{\alpha}\right\}_{\alpha \in I}$ on $X$ by $f_{\alpha}(x)=\prod_{i \in S} x_{i}^{\alpha}{ }_{i}$. Then we have by Lemma 2.3 in $[10]$ the following

Lemma 2.1. Let $\mu \in \mathscr{P}(X)$ and $f(\alpha)=\left\langle\mu, f_{\alpha}\right\rangle$. Then it holds that

$$
\left\langle T_{t}^{*} \mu, f_{\alpha}\right\rangle=\boldsymbol{E}_{\alpha}\left[f\left(\alpha_{t}\right)\right] \quad \text { for each } \alpha \in I \text {. }
$$

Let us introduce some kind of Markov times.

$$
\begin{aligned}
& \zeta=\inf \left\{t \geqq 0 ;\left|\alpha_{t}\right|<\left|\alpha_{0}\right|\right\}, \zeta_{k}=\inf \left\{t \geqq 0 ;\left|\alpha_{t}\right| \leqq k\right\} \quad \text { for } k \geqq 0, \\
& \tau=\inf \left\{t \geqq 0 ; \alpha_{t} \in I \backslash \Delta \text { and }\left|\alpha_{t}\right|=\left|\alpha_{0}\right|\right\}, \sigma=\inf \left\{t \geqq 0 ; \alpha_{t} \in \Delta\right\},
\end{aligned}
$$

and $\tau_{n}$ and $\sigma_{n}$ are defined inductively by

$$
\tau_{n}=\sigma_{n-1}+\tau\left(\vartheta_{\tau_{n-1}}\right) \text { and } \sigma_{n}=\tau_{n}+\sigma\left(\vartheta_{\tau_{n}}\right), \quad \text { where } \sigma_{0}=0 .
$$

Let us denote $\Omega_{1}=\left[\int_{0}^{\infty} I_{\Delta}\left(\alpha_{t}\right) d t=+\infty\right]$ and $\Omega_{0}=\left[\int_{0}^{\infty} I_{\Delta}\left(\alpha_{t}\right) d t<+\infty\right]$. Then the following lemma holds.

Lemma 2.2. Let $g$ be any bounded function on $I$. Then,

(1) $\boldsymbol{E}_{\alpha}\left[g\left(\alpha_{t}\right) ; t<\sigma_{1}\right]=\tilde{\boldsymbol{E}}_{\alpha}\left[g\left(\alpha_{t}\right) ; t<\sigma_{1}\right] \quad$ if $\alpha \in I \backslash \Delta$,

(2) $\left[\tau_{n}<+\infty, \sigma_{n}=+\infty\right.$ for some $\left.n \geqq 1\right]=\Omega_{0}$

a.s. $\left(\tilde{\boldsymbol{P}}_{\alpha}\right)$,

(3) $[\zeta=+\infty]=\left[\tau_{n}<+\infty, \sigma_{n}=+\infty\right.$ for some $\left.n \geqq 1\right]$

a.s. $\left(\boldsymbol{P}_{\alpha}\right)$,

(4) $\lim _{t \rightarrow \infty} \boldsymbol{E}_{\alpha}\left[\tilde{\boldsymbol{P}}_{\alpha_{t}}\left[\Omega_{1}\right]\right]=0$,

$\langle\mu, f\rangle=\int_{X^{\prime}} \mu(d x) f(x)$

$\boldsymbol{E}_{\alpha}$ denotes the exectation by $\boldsymbol{P}_{\alpha}$. 
(5) $\left|\boldsymbol{E}_{\alpha}\left[g\left(\alpha_{t}\right)\right]-\tilde{\boldsymbol{E}}_{\alpha}\left[g\left(\alpha_{t}\right)\right]\right| \leqq\|g\| \boldsymbol{P}_{\alpha}[\zeta<+\infty] \leqq\|g\| \tilde{\boldsymbol{P}}_{\alpha}\left[\sigma_{1}<+\infty\right]$,

and

(6) $\lim _{t \rightarrow \infty} \mid \boldsymbol{E}_{\varepsilon i+\varepsilon j}\left[g\left(\alpha_{t}\right)\right]-\boldsymbol{E}_{\varepsilon}\left[\left[g\left(\alpha_{t}\right)\right] \mid \leqq\|g\| \boldsymbol{P}_{\varepsilon i+s j}[\zeta=+\infty] \leqq\|g\| \tilde{\boldsymbol{P}}_{\varepsilon i+s j}\left[\Omega_{0}\right]\right.$.

Proof. We shall show only (4), since (1) (3) are trivial and (5) and (6) can be shown by constructing coupling processes. (cf. Lemma 4.9 in [10]).

For $\alpha \in I \backslash \Delta$

$$
\begin{aligned}
& \lim _{t \rightarrow \infty} \boldsymbol{E}_{\alpha}\left[\tilde{\boldsymbol{P}}_{\alpha_{t}}\left[\Omega_{1}\right] ; \sigma_{1}=+\infty\right]=\lim _{t \rightarrow \infty} \tilde{\boldsymbol{E}}_{\alpha}\left[\tilde{\boldsymbol{P}}_{\alpha_{t}}\left[\Omega_{1}\right] ; \sigma_{1}>t\right] \\
= & \lim _{t \rightarrow \infty} \tilde{\boldsymbol{P}}_{\alpha}\left[\Omega_{1} \cap\left[\sigma_{1}>t\right]\right]=\tilde{\boldsymbol{P}}_{\alpha}\left[\Omega_{1} \cap\left[\sigma_{1}=+\infty\right]\right]=0 .
\end{aligned}
$$

Also,

$$
\begin{aligned}
& \lim _{t \rightarrow \infty} \boldsymbol{E}_{\alpha}\left[\tilde{\boldsymbol{P}}_{\alpha_{t}}\left[\Omega_{1}\right] ; \zeta=+\infty\right]=\lim _{t \rightarrow \infty} \sum_{n=1}^{\infty} \boldsymbol{E}_{\alpha}\left[\tilde{\boldsymbol{P}}_{\alpha_{t}}\left[\Omega_{1}\right] ; \tau_{n}<t, \sigma_{n}=+\infty\right] \\
= & \lim _{t \rightarrow \infty} \sum_{n=1}^{\infty} \boldsymbol{E}_{\alpha}\left[\left.\boldsymbol{E}_{\alpha_{\tau_{n}}}\left[\tilde{\boldsymbol{P}}_{\alpha_{u-t}}\left[\Omega_{1}\right] ; \sigma_{1}=+\infty\right]\right|_{u=\tau_{n}} ; \tau_{n}<+\infty\right] \\
= & \sum_{n=1}^{\infty} \boldsymbol{E}_{\alpha}\left[\lim _{t \rightarrow \infty} \boldsymbol{E}_{\alpha_{\tau_{n}}}\left[\tilde{\boldsymbol{P}}_{\alpha_{t}}\left[\Omega_{1}\right] ; \sigma_{1}=+\infty\right] ; \tau_{n}<+\infty\right]=0, \quad \text { by }(2.4) .
\end{aligned}
$$

Thus, we have

$$
\begin{aligned}
\lim _{t \rightarrow \infty} \boldsymbol{E}_{\alpha}\left[\tilde{\boldsymbol{P}}_{\alpha_{t}}\left[\Omega_{1}\right]\right] & =\lim _{t \rightarrow \infty} \sum_{k=2}^{|\alpha|} \boldsymbol{E}_{\alpha}\left[\tilde{\boldsymbol{P}}_{\alpha_{t}}\left[\Omega_{1}\right] ; \zeta_{k}<t, \zeta_{k-1}=+\infty\right] \\
& =\sum_{k=2}^{|\alpha|} \boldsymbol{E}_{\alpha}\left[\lim _{s \rightarrow \infty} \boldsymbol{E}_{\alpha \zeta_{k}}\left[\tilde{\boldsymbol{P}}_{\alpha_{s}}\left[\Omega_{1}\right] ; \zeta=+\infty\right] ; \zeta_{k}<+\infty\right]=0 .
\end{aligned}
$$

Let us define the spaces of harmonic functions of $\left(\alpha_{\ell}, \tilde{\boldsymbol{P}}_{\alpha}\right)_{\alpha \in I}$, and $\left(\alpha_{t}, \boldsymbol{P}_{\alpha}\right)_{\alpha \in I}$.

$$
\begin{aligned}
& \tilde{\mathscr{C}}=\left\{\tilde{h} ; \text { defined on } I, 0 \leqq \tilde{h} \leqq 1 \text {, and } \tilde{\boldsymbol{E}}_{\alpha}\left[\tilde{h}\left(\alpha_{t}\right)\right]=\tilde{h}(\alpha)\right. \\
& \text { for each } \alpha \in I \text { and } t \geqq 0\} \text {, } \\
& \mathcal{G}=\left\{g \text {; defined on } I, 0 \leqq g \leqq 1 \text {, and } \boldsymbol{E}_{\alpha}\left[g\left(\alpha_{t}\right)\right]=g(\alpha)\right. \\
& \text { for each } \alpha \in I \text { and } t \geqq 0\} \text {. }
\end{aligned}
$$

Then we can obtain the following lemma by a similar argument to Lemma 4.2 , Lemma 4.3, and Lemma 4.4 in [10].

\section{Lemma 2.3.}

(1) For each $\tilde{h} \in \widetilde{\mathscr{H}} \lim _{t \rightarrow \infty} \boldsymbol{E}_{\alpha}\left[\tilde{h}\left(\alpha_{t}\right)\right]$ exists for each $\alpha \in I$, and

(2) for each $g \in \mathcal{G} \lim _{t \rightarrow \infty} \tilde{\boldsymbol{E}}_{\alpha}\left[g\left(\alpha_{t}\right) ; \Omega_{0}\right]$ exists for each $\alpha \in I$.

So, denoting $\Phi \tilde{h}(\alpha)=\lim _{t \rightarrow \infty} \boldsymbol{E}_{\alpha}\left[\tilde{h}\left(\alpha_{t}\right)\right]$ and $\Psi_{1} g(\alpha)=\lim _{t \rightarrow \infty} \tilde{\boldsymbol{E}}_{\alpha}\left[g\left(\alpha_{t}\right) ; \Omega_{0}\right]$, $\Phi$ is a map from $\widetilde{\mathscr{H}}$ into $\mathcal{G}$, and $\Psi_{1}$ is a map from $\mathcal{G}$ into $\widetilde{\mathcal{H}}$. Moreover, it holds that 
(3) $\Phi \Psi_{1} g=g$ for each $g \in \mathcal{G}$, and

(4) $\Psi_{1} \Phi \tilde{h}(\alpha)=\lim _{t \rightarrow \infty} \tilde{\boldsymbol{E}}_{\alpha}\left[\tilde{h}\left(\alpha_{t}\right) ; \Omega_{0}\right]$ for each $\tilde{h} \in \tilde{\mathscr{H}}$ and $\alpha \in I$.

Lemma 2.4 (Lemma 4.8 in [10]). Let $f_{i, j}^{*}=\tilde{\boldsymbol{P}}_{\varepsilon i+\varepsilon j}\left[\Omega_{0} \cap\left[\sigma_{1}<+\infty\right]\right]$. Then,

$$
\lim _{t \rightarrow \infty} \sum_{k \in S} P_{t}(i, k) f_{k, j}^{*}=0 \quad \text { for each }(i, j) \in S \times S .
$$

Lemma 2.5. Let $f_{i, j}=\tilde{\boldsymbol{P}}_{\varepsilon i+\varepsilon j}\left[\sigma_{1}<+\infty\right], \quad \bar{f}_{i, j}=\tilde{\boldsymbol{P}}_{i_{i+\varepsilon} j}\left[\Omega_{1}\right]$, and $F_{i, j}=f_{i, j} \wedge$ $\left(1-\bar{f}_{i, j}\right)$. Then,

$$
\lim _{t \rightarrow \infty} \varlimsup_{s \rightarrow \infty} \sum_{k \in S} \sum_{m \in S} P_{t}(i, k) P_{s}(j, m) F_{k, m}=0 .
$$

Proof. Noting $f_{i, j}=f_{i, j}^{*}+\bar{f}_{i, j}, F_{i, j} \leqq f_{i, j}^{*}+\bar{f}_{i, j} \wedge\left(1-\bar{f}_{i, j}\right)$ and Lemma 2.4, it suffice to show

$$
\lim _{t, s \rightarrow \infty} \sum_{k \in S} \sum_{m \in S} P_{t}(i, k) P_{s}(j, m) \bar{f}_{k, m} \wedge\left(1-\bar{f}_{k, m}\right)=0 .
$$

Let $\left(\left(X_{t}^{1}, X_{t}^{2}\right), P_{(i, j)}^{(2)}\right)$ be a continuous time Markov chain corresponding to the transition probability $P_{t} \otimes P_{t}$. Let us denote by $\mathscr{B}_{t, s}$ the $\sigma$-field generated by $\left\{\left(X_{u}^{1}, X_{v}^{2}\right) ; 0 \leqq u \leqq t, 0 \leqq v \leqq s\right\}$ and

$$
\Omega^{(1)}=\left[\int_{0}^{\infty} I_{\Delta_{2}}\left(X_{t}^{1}, X_{t}^{2}\right) d t=+\infty\right] .
$$

First we claim that

$$
\bar{f}_{X_{t}^{1}, X_{s}^{2}}=P_{(i, j)}^{(2)}\left[\Omega^{(1)} \mid \mathscr{B}_{t, s}\right] \text { a.s. }\left(P_{(i, j)}^{(2)}\right) \quad \text { for all } t, s \geqq 0 .
$$

Since $\Omega^{(1)}$ is a tail event, by the Markov property we have

$$
P_{(i, j)}^{(2)}\left[\Omega^{(1)} \mid \mathscr{B}_{t, t}\right]=P_{\left(X_{t}^{1}, X_{t}^{2}\right)}^{(2)}\left[\Omega^{(1)}\right]=\bar{f}_{X_{t}^{1}, X_{t}^{2}} \quad \text { for each } t \geqq 0 .
$$

Also, we may assume $t \geqq s$, and then it follows that

$$
\begin{aligned}
& P_{(i, j)}^{(2)} {\left[\Omega^{(1)} \mid \mathscr{B}_{t, s}\right]=E_{(i, j)}^{(2)}\left[P_{(i, j)}^{(2)}\left[\Omega^{(1)} \mid \mathscr{B}_{t, t}\right] \mid \mathscr{B}_{t, s}\right] } \\
& \quad=E_{(i, j)}^{(2)}\left[\bar{f}_{X_{t}^{1}, X_{t}^{2}} \mid \mathscr{B}_{t, s}\right]=\sum_{j \in S} P_{t-s}\left(X_{s}^{2}, j\right) \bar{f}_{X_{t}^{1}, j}=\bar{f}_{X_{t}^{1}, X_{s}^{2}} .
\end{aligned}
$$

Here we used a fact that $\bar{f}_{i, j}$ is $P_{t}$-harmonic function of each variable by Lemma 4.7 in [10], since $\bar{f}_{i, j}$ is $P_{t} \otimes P_{t}$-harmonic. For any $\varepsilon>0$, there exist $t_{0}>0, s_{0}>0$ and an event $\Omega_{1}^{*}$ such that

$$
\Omega_{1}^{*} \in \mathscr{B}_{t_{0}, s_{0}} \quad \text { and } \quad P_{(i, j)}^{(2)}\left[\Omega^{(1)} \ominus \Omega_{1}^{*}\right]<\varepsilon .
$$

Then for $t>t_{0}$ and $s>s_{0}$,

$$
\begin{aligned}
& E_{(i, j)}^{(2)}\left[\left|\bar{f}_{X_{t}^{1} X_{s}^{2}}-I_{\Omega^{(1)}}\right|\right]=E_{(i, j)}^{(2)}\left[\left|P_{(i, j)}^{(2)}\left[\Omega^{(1)} \mid \mathscr{B}_{t, s}\right]-I_{\Omega^{(1)}}\right|\right] \\
& \leqq E_{(i, j)}^{(2)}\left[P_{(i, j)}^{(2)}\left[\Omega^{(1)} \ominus \Omega_{1}^{*} \mid \mathscr{B}_{t, s}\right]\right]+P_{(i, j)}^{(2)}\left[\Omega^{(1)} \ominus \Omega_{1}^{*}\right]<2 \varepsilon,
\end{aligned}
$$

$a \wedge b=\min \{a, b\}$. 
because of $P_{(i, j)}^{(2)}\left[\Omega_{1}^{*} \mid \mathscr{B}_{t, s}\right]=I_{\Omega_{1}^{*}}$.

$$
\begin{aligned}
E_{(i, j)}^{(2)}\left[\bar{f}_{X_{t}^{1}, X_{s}^{2}} \wedge\left(1-\bar{f}_{\left.X_{t}^{1}, X_{s}^{2}\right)}\right]\right. & =E_{(i, j)}^{(2)}\left[\bar{f}_{X_{t}^{1}, X_{s}^{2}} \wedge\left(1-\bar{f}_{X_{t}^{1}, X_{s}^{2}}\right)-I_{\Omega^{(1)}} \wedge\left(1-I_{\Omega^{(1)}}\right)\right] \\
& \leqq 2 E_{(i, j)}^{(2)}\left[\left|\bar{f}_{X_{t}^{1}, X_{s}^{2}}-I_{\Omega^{(1)}}\right|\right]<4 \varepsilon
\end{aligned}
$$

for all $t>t_{0}$ and $s>s_{0}$. Thus, we get (2.8).

The following lemma holds in the same way as the voter model.

Lemma 2.6. Let $\mu \in \mathcal{S}_{e x}, g(\alpha)=\left\langle\mu, f_{a}\right\rangle$ and $h(i)=g\left(\varepsilon^{i}\right)$. Then.

(1) $\left|g\left(\varepsilon^{i}+\varepsilon^{j}\right)-h(i) h(j)\right| \leqq 2 F_{i, j}$, and

(2) $h \in \mathscr{A}^{*}$.

Proof. First, we claim that

$$
\left|\tilde{\boldsymbol{E}}_{\varepsilon^{i}+\varepsilon j}\left[g\left(\alpha_{t}\right)\right]-g\left(\varepsilon^{i}+\varepsilon^{j}\right)\right| \leqq 2 F_{i, j} .
$$

By Lemma 2.2 (6), we have

$$
\begin{aligned}
\left|g\left(\varepsilon^{i}+\varepsilon^{j}\right)-h(i)\right| & =\left|\boldsymbol{E}_{\varepsilon i+\varepsilon j}\left[g\left(\alpha_{t}\right)\right]-\boldsymbol{E}_{\varepsilon i}\left[g\left(\alpha_{t}\right)\right]\right| \\
& \leqq \tilde{\boldsymbol{P}}_{\varepsilon^{i}+\varepsilon j}\left[\Omega_{0}\right]=1-\bar{f}_{i, j} .
\end{aligned}
$$

Also,

$$
\begin{aligned}
\left|\tilde{\boldsymbol{E}}_{\varepsilon i+s} j\left[g\left(\alpha_{t}\right)\right]-h(i)\right| & \leqq\left|\sum_{k \in S} \sum_{m \in S} P_{\iota}(i, k) P_{t}(j, m)\left(g\left(\varepsilon^{k}+\varepsilon^{m}\right)-h(k)\right)\right| \\
& \leqq \sum_{k \in S} \sum_{m \in S} P_{t}(i, k) P_{t}(j, m)\left(1-\bar{f}_{k, m}\right)=1-\bar{f}_{i, j}
\end{aligned}
$$

Thus, we have

$$
\left|\tilde{\boldsymbol{E}}_{\varepsilon i+\varepsilon j}\left[g\left(\alpha_{t}\right)\right]-g\left(\varepsilon^{i}+\varepsilon^{j}\right)\right| \leqq 2\left(1-\bar{f}_{i, j}\right) .
$$

On the other hand by Lemma 2.2 (5),

$$
\begin{aligned}
\left|\tilde{\boldsymbol{E}}_{i^{i}+\varepsilon} j\left[g\left(\alpha_{t}\right)\right]-g\left(\varepsilon^{i}+\varepsilon^{j}\right)\right| & =\mid \tilde{\boldsymbol{E}}_{\varepsilon i+\varepsilon j}\left[g\left(\alpha_{t}\right)-\boldsymbol{E}_{\varepsilon i+\varepsilon j}\left[g\left(\alpha_{t}\right)\right] \mid\right. \\
& \leqq \boldsymbol{P}_{\varepsilon i_{+} j}\left[\sigma_{1}<+\infty\right]=f_{i, j} .
\end{aligned}
$$

Hence (2.10) follows from (2.12) and (2.13).

Next, it is easy to see that $\lim _{s \rightarrow \infty} \sum_{k \in S} \sum_{m \in S} P_{s}(i, k) P_{s}(j, m) f_{k, m}^{*}=0$, so we have by (2.8)

$$
\lim _{s \rightarrow \infty} \sum_{k \in S} \sum_{m \in S} P_{s}(i, k) P_{s}(j, m) F_{k, m}=0 .
$$

Accordingly, it follows from (2.10) and (2.14) that $\lim _{t \rightarrow \infty} \tilde{\boldsymbol{E}}_{\varepsilon^{i}+\varepsilon j}\left[g\left(\alpha_{t}\right)\right]$ exists, which is denoted by $h(i, j)$.

Thus, we have

$$
\left|g\left(\varepsilon^{i}+\varepsilon^{j}\right)-h(i, j)\right| \leqq 2 F_{i, j} .
$$

Finally we claim that $h(i, j)=h(i) h(j)$ for all $i$ and $j$. Since $\mu \in \mathcal{S}_{e x}$, it follows from (4.16) in [10] that 


$$
\lim _{T \rightarrow \infty} \frac{1}{T} \int_{0}^{T} \sum_{k \in s} P_{t}(i, k) g\left(\varepsilon^{k}+\varepsilon^{j}\right) d t=g\left(\varepsilon^{i}\right) g\left(\varepsilon^{j}\right)=h(i) h(j) .
$$

Noting that $h(i, j)$ is $P_{t}$-harmonic in each variable, and combining (2.12), (2.16) and Lemma 2.5, we can see that $h(i, j)=h(i) h(j)$ for all $i$ and $j$.

Next, we shall show $h \in \mathscr{H}^{*}$. By (2.11),

$$
|h(i) h(j)-h(i)| \leqq 1-\bar{f}_{i, j} \quad(i, j \in S) .
$$

Then it is easy to see that

$$
\lim _{t \rightarrow \infty} h\left(X_{t}^{1}\right)=\lim _{t \rightarrow \infty} h\left(X_{t}^{2}\right)=0 \text { or } 1 \quad \text { a.s. }\left(P_{(i, j)}^{(2)}\right) \text { on }\left[\int_{0}^{\infty} I_{\Delta_{2}}\left(X_{t}\right) d t=+\infty\right] .
$$

Thus, we complete the proof of Lemma 2.6.

\section{Proof of Theorems}

We shall begin with the proof of Theorem 1.2. Assume that $\lim _{t \rightarrow \infty} T_{t}^{*} \mu=\nu_{h}$ for some $h \in \mathscr{H}^{*}$.

Let $\left\langle\mu, f_{\alpha}\right\rangle=g(\alpha)$ and $\left\langle\nu_{h}, f_{\alpha}\right\rangle=g^{h}(\alpha)$. Then it holds that

$$
\lim _{t \rightarrow \infty} E_{\alpha}\left[g\left(\alpha_{t}\right)\right]=g^{n}(\alpha) \quad \text { for each } \alpha \in I,
$$

and particularly

$$
\lim _{t \rightarrow \infty} \sum_{j \in S} P_{t}(i, j) g\left(\varepsilon^{j}\right)=h(i) \quad \text { for each } i \in S .
$$

First, we claim that

$$
\lim _{t \rightarrow \infty} \tilde{\boldsymbol{E}}_{\varepsilon i+\varepsilon j}\left[g\left(\alpha_{t}\right)\right]=h(i) h(j) \quad \text { for each }(i, j) \in S \times S .
$$

For this it is sufficient to show that if for a sequence $\left\{t_{n}\right\}$ tending to $+\infty$, $\lim _{n \rightarrow \infty} \tilde{\boldsymbol{E}}_{\alpha}\left[g\left(\alpha_{t_{n}}\right)\right]$ exists (which is denoted by $\bar{h}(\alpha)$ ) for each $\alpha \in I$, then $\bar{h}\left(\varepsilon^{i}+\varepsilon^{j}\right)=$ $h(i) h(j)$ holds for each $i, j \in S$. Evidently it holds that

$$
\begin{aligned}
\bar{h}\left(\varepsilon^{i}+\varepsilon^{j}\right) & =\lim _{n \rightarrow \infty} \sum_{k \in S} \sum_{m \in S} P_{t_{n}}(i, k) P_{t_{n}}(j, m) g\left(\varepsilon^{k}+\varepsilon^{m}\right) \\
& \leqq \lim _{n \rightarrow \infty} \sum_{k \in S} P_{t_{n}}(i, k) g\left(\varepsilon^{k}\right)=h(i), \quad \text { and } \\
\bar{h}\left(2 \varepsilon^{i}\right)-h(i)^{2} & =\lim _{n \rightarrow \infty} \sum_{k \in S} \sum_{m \in S} P_{t_{n}}(i, k) P_{t_{n}}(i, m) g\left(\varepsilon^{k}+\varepsilon^{m}\right)-h(i)^{2} \\
& =\lim _{n \rightarrow \infty} \int_{X}\left(\sum_{k \in S} P_{t_{n}}(i, k) x_{k}-h(i)\right)^{2} \mu(d x) \geqq 0
\end{aligned}
$$

Also, it follows that for any $\alpha \in I$,

$$
\lim _{t \rightarrow \infty} \tilde{\boldsymbol{E}}_{\alpha}\left[\bar{h}\left(\alpha_{t}\right) ; \sigma_{1}=+\infty\right]=\lim _{t \rightarrow \infty} \tilde{\boldsymbol{E}}_{\alpha}\left[\tilde{h}\left(\alpha_{t}\right) ; \sigma_{1}=+\infty\right],
$$

where $\tilde{h}(\alpha)=\prod_{i \in S} h(i)^{\alpha}$. 
For

$$
\begin{aligned}
& \lim _{t \rightarrow \infty} \tilde{\boldsymbol{E}}_{\alpha}\left[\bar{h}\left(\alpha_{t}\right) ; \sigma_{1}=+\infty\right]=\lim _{t \rightarrow \infty} \lim _{n \rightarrow \infty} \tilde{\boldsymbol{E}}_{\alpha}\left[\tilde{\boldsymbol{E}}_{\alpha_{t}}\left[g\left(\alpha_{t_{n}}\right)\right] ; \sigma_{1}=+\infty\right] \\
= & \lim _{t \rightarrow \infty} \lim _{n \rightarrow \infty} \tilde{\boldsymbol{E}}_{\alpha}\left[\tilde{\boldsymbol{E}}_{\alpha_{t}}\left[g\left(\alpha_{t_{n}}\right)\right] ; \sigma_{1}>t\right]=\lim _{t \rightarrow \infty} \lim _{n \rightarrow \infty} \tilde{\boldsymbol{E}}_{\alpha}\left[g\left(\alpha_{t_{n}+t}\right) ; \sigma_{1}>t\right] \\
= & \lim _{t \rightarrow \infty} \boldsymbol{E}_{\alpha}\left[g\left(\alpha_{t}\right) ; \sigma_{1}=+\infty\right]=\lim _{t \rightarrow \infty} \boldsymbol{E}_{\alpha}\left[g\left(\alpha_{t}\right)\right]-\lim _{t \rightarrow \infty} \boldsymbol{E}_{\alpha}\left[g\left(\alpha_{t}\right) ; \sigma_{1}<\infty\right] \\
= & g^{h}(\alpha)-\boldsymbol{E}_{\alpha}\left[g^{h}\left(\alpha_{\sigma_{1}}\right) ; \sigma_{1}<+\infty\right]=\lim _{t \rightarrow \infty} \tilde{\boldsymbol{E}}_{\alpha}\left[\tilde{h}\left(\alpha_{t}\right) ; \sigma_{1}=+\infty\right] .
\end{aligned}
$$

Thus, using (3.6) we can see easily that

$$
\lim _{t \rightarrow \infty} \tilde{\boldsymbol{E}}_{\alpha}\left[\bar{h}\left(\alpha_{t}\right) ; \Omega_{0}\right]=\lim _{t \rightarrow \infty} \tilde{\boldsymbol{E}}_{\alpha}\left[\tilde{h}\left(\alpha_{t}\right) ; \Omega_{0}\right] \quad \text { for } \alpha \in I .
$$

Next we shall show

$$
\lim _{t \rightarrow \infty} \tilde{\boldsymbol{E}}_{\varepsilon^{i}+\varepsilon j}\left[\bar{h}\left(\alpha_{t}\right) ; \Omega_{1}\right]=\lim _{t \rightarrow \infty} \tilde{\boldsymbol{E}}_{\varepsilon^{i+\varepsilon}+j}\left[\tilde{h}\left(\alpha_{t}\right) ; \Omega_{1}\right] \quad \alpha \in I .
$$

Since $\Omega_{1}=\left[\sigma_{n}<+\infty\right.$ for all $\left.n\right]$ a.s. $\left(\tilde{\boldsymbol{P}}_{\alpha}\right)$, it follows from (3.5),

$$
\begin{aligned}
& \lim _{t \rightarrow \infty} \tilde{\boldsymbol{E}}_{i^{i+\varepsilon}}\left[\bar{h}\left(\alpha_{t}\right) ; \Omega_{1}\right]=\lim _{n \rightarrow \infty} \tilde{\boldsymbol{E}}_{\varepsilon^{i+\varepsilon}}\left[\bar{h}\left(\alpha_{\sigma_{n}}\right) ; \Omega_{1}\right] \\
& \geqq \lim _{n \rightarrow \infty} \tilde{\boldsymbol{E}}_{\varepsilon^{i}+\varepsilon j}\left[\tilde{h}\left(\alpha_{\sigma_{n}}\right) ; \Omega_{1}\right]=\lim _{t \rightarrow \infty} \tilde{\boldsymbol{E}}_{\varepsilon^{i+\varepsilon} j}\left[\tilde{h}\left(\alpha_{t}\right) ; \Omega_{1}\right] .
\end{aligned}
$$

On the other hand if we identify $\left(\alpha_{t}, \tilde{\boldsymbol{P}}_{\varepsilon i+\varepsilon j}\right)$ with $\left(\left(X_{t}^{1}, X_{t}^{2}\right), P_{(i, j)}^{(2)}\right)$,

$$
\begin{aligned}
\lim _{t \rightarrow \infty} \tilde{\boldsymbol{E}}_{\varepsilon^{i}+\varepsilon j}\left[\bar{h}\left(\alpha_{t}\right) ; \Omega_{1}\right] & =\lim _{t \rightarrow \infty} E_{(i, j)}^{(2)}\left[\bar{h}\left(\varepsilon_{X_{t}^{1}}+\varepsilon_{X_{t}^{2}}\right) ; \Omega^{(1)}\right] \\
& \leqq \lim _{t \rightarrow \infty} E_{(i, j)}^{(2)}\left[h\left(X_{t}^{1}\right) ; \Omega^{(1)}\right] \quad(\text { by }(3.4)) \\
& =\lim _{t \rightarrow \infty} E_{(i, j)}^{(2)}\left[h\left(X_{t}^{1}\right) h\left(X_{t}^{2}\right) ; \Omega^{(1)}\right] \\
& =\lim _{t \rightarrow \infty} \tilde{\boldsymbol{E}}_{\varepsilon i+\varepsilon j}\left[\tilde{h}\left(\alpha_{t}\right) ; \Omega_{1}\right] \quad \text { holds. }
\end{aligned}
$$

Thus we get (3.8).

Moreover we note that $\bar{h} \in \tilde{\mathscr{T}}$ by Lemma 4.5 in [10]. Hence we can conclude by (3.7) and (3.8) that

$$
\bar{h}\left(\varepsilon^{i}+\varepsilon^{j}\right)=\tilde{h}\left(\varepsilon^{i}+\varepsilon^{j}\right)=h(i) h(j) \quad \text { holds for all } i \text { and } j .
$$

By (3.2) and (3.3), it follows obviously that

$$
\lim _{t \rightarrow \infty} \int_{X}\left(\sum_{j \in S} P_{t}(i, j) x_{j}-h(i)\right)^{2} \mu(d x)=0,
$$

and this implies (1.7).

The proof of the converse is easy and it is found in $\S 5$ of [10].

Next, we shall prove Theorem 1.1.

By Lemma 2.3, it is immediate that for each $h \in \mathscr{H} \lim _{t \rightarrow \infty} T_{t}^{*} \delta_{h}=\nu_{h}$ exists and $\int_{X} x_{i} \nu_{h}(d x)=h(i)$ holds for each $i \in S$.

First we shall show that $\left\{\nu_{h} ; h \in \mathscr{H}^{*}\right\} \subset \mathcal{S}_{e x}$ holds. 
Let $h \in \mathscr{H}^{*}$, and assume $\nu_{h}=(1 / 2)\left(\mu^{\prime}+\mu^{\prime \prime}\right)$ with $\mu^{\prime}$ and $\mu^{\prime \prime} \in \mathcal{S}$. Theorem 1.2 implies that $\sum_{j \in S} P_{t}(i, j) x_{j}$ converges to $h(i)$ in probablity with respect to $\nu_{h}$, and also it is true for $\mu^{\prime}$ and $\mu^{\prime \prime}$. Again by Theorem 1.2 we have $\mu^{\prime \prime}=\lim _{t \rightarrow \infty} T_{t}^{*} \mu^{\prime}=\nu_{h}$. Hence $\nu_{h}$ is extremal.

Conversely let $\mu \in \mathcal{S}_{e x}, g(\alpha)=\left\langle\mu, f_{\alpha}\right\rangle$ and $h(i)=g\left(\varepsilon^{i}\right)$. Then Lemma 2.6 and (2.14) imply that (3.9) holds. Hence,

$$
\lim _{t \rightarrow \infty} \tilde{\boldsymbol{E}}_{\alpha}\left[g\left(\alpha_{t}\right)\right]=\tilde{h}(\alpha) \quad \text { for each } \alpha \in I .
$$

By Lemma 2.3, (3.10) and Lemma 2.2, we have

$$
\begin{aligned}
\left|\left\langle\nu_{h}, f_{\alpha}\right\rangle-g(\alpha)\right| & =|\Phi \tilde{h}(\alpha)-g(\alpha)| \\
& =\left|\Phi \Psi_{1} g(\alpha)+\lim _{t \rightarrow \infty} \lim _{s \rightarrow \infty} \boldsymbol{E}_{\alpha}\left[\tilde{\boldsymbol{E}}_{\alpha_{t}}\left[g\left(\alpha_{s}\right) ; \Omega_{1}\right]\right]-g(\alpha)\right| \\
& \leqq \lim _{t \rightarrow \infty} \boldsymbol{E}_{\alpha}\left[\tilde{\boldsymbol{P}}_{\alpha_{t}}\left[\Omega_{1}\right]\right]=0 \quad \text { for each } \alpha \in I .
\end{aligned}
$$

Therefore $\mu=\nu_{h}$ holds, and $h \in \mathscr{H}^{*}$ follows from Lemma 2.6 .

\section{Examples}

In this section we shall present two examples of Case III.

$\mathbf{1}^{\circ}$ Consider the example in $\S 6$ of $[10]$ with $k=+\infty$.

Let $S=\{s=(i, n) ; i \in N, n \in N\} \cup\{0\}$, and $Q=\left\{q_{s, s^{\prime}}\right\}$ be given by $q_{0,(i, 1)}=a_{i}$ $>0, \quad q_{0,0}=-\sum_{i=1}^{\infty} a_{i}, q_{(i, n),(i, n+1)}=\lambda_{n}>0, \quad q_{(i, n),(i, n-1)}=\mu_{n}>0 \quad(n \geqq 1), \quad q_{(i, n),(i, n)}=$ $-\left(\lambda_{n}+\mu_{n}\right)$, and $q_{s, s^{\prime}}=0$ for all other $\left(s, s^{\prime}\right)$.

Assume that $\sup _{n}\left(\lambda_{n}+\mu_{n}\right)<+\infty, \sum_{i=1}^{\infty} a_{i}<+\infty$, and that the continuous time Markov chain $\left(X_{t}, P_{s}\right)_{s \in S}$, generated by $Q=\left\{q_{s, s^{\prime}}\right\}$, is transient. Then, $Q$ satisfies the condition of Case III. Denote by $\left\{i^{\infty} ; i \in N\right\}$ the set of infinity points of $S$, and for each subset $\beta \subset N$, define $h_{\beta}(s)=P_{s}\left[\lim _{t \rightarrow \infty} X_{t} \in \beta^{\infty}\right]$, where $\beta^{\infty}=\left\{i^{\infty} ; i \in \beta\right\}$.

Then it is easy to check that $\Omega^{(1)}=\left[\lim _{t \rightarrow \infty} X_{t}^{1}=\lim _{t \rightarrow \infty} X_{t}^{2}=i^{\infty}\right.$ for some $\left.i\right]$ holds. So, we have $\mathscr{H}_{e x}=\mathscr{H}^{*}=\left\{h_{\beta} ; \beta \subset N\right\}$.

$2^{\circ} \quad$ Next, we shall consider an example such that $\mathscr{H}_{e x} \subsetneq \mathscr{H}^{*}$ holds.

Let $S=Z^{3} \cup\{0,1,2, \cdots\}, Q=\left\{q_{i, j}\right\}$ is given as follows.

Let $i=\left(i_{1}, i_{2}, i_{3}\right) \in \boldsymbol{Z}^{3}$.

$$
q_{i, j}= \begin{cases}1 & \text { if } i_{3}=0 \text { and } j=\left(i_{1}, i_{2}, \pm 1\right), \text { or } j=\left(j_{1}, j_{2}, i_{3}\right) \text { with }|j-i|=1 . \\ p & \text { if } j=\left(i_{1}, i_{2}, i_{3}+1\right) \text { with } i_{3}>0, \text { or } j=\left(i_{1}, i_{2}, i_{3}-1\right) \text { with } i_{3}<0, \\ q & \text { if } j=\left(i_{1}, i_{2}, i_{3}-1\right) \text { with } i_{3}>0, \text { or } j=\left(i_{1}, i_{2}, i_{3}+1\right) \text { with } i_{3}<0,\end{cases}
$$

$q_{(0,0,0), 0}=q_{0,(0,0,0)}=1, q_{n, n+1}=p, q_{n, n-1}=q(n \geqq 1)$ and $q_{i, j}=0$ for all other $(i, j) \in$

$\boldsymbol{N}$ denotes the set of natural numbers. 
$S \times S$ with $i \neq j$. We assume $p>q>0$. Then $Q=\left\{q_{i, j}\right\}$ satisfies the condition of Case III. Let $L^{+}=\left\{i=\left(i_{1}, i_{2}, i_{3}\right) \in \boldsymbol{Z}^{3} ; i_{3}>0\right\}, L^{-}=\left\{i=\left(i_{1}, i_{2}, i_{3}\right) \in \boldsymbol{Z}^{3} ; i_{3}<0\right\}$, and $L_{0}$ $=\{0,1,2, \cdots\}$. Let us define $h^{+}(i)=P_{i}\left[\int_{0}^{\infty} I_{L^{+}}\left(X_{t}\right) d t=+\infty\right], h^{-}(i)=P_{i}\left[\int_{0}^{\infty} I_{L^{-}}\left(X_{t}\right) d t\right.$ $=+\infty]$ and $h_{0}(i)=P_{i}\left[\int_{0}^{\infty} I_{L_{0}}\left(X_{t}\right) d t=+\infty\right]$. Then we have

$$
\mathscr{H}^{*}=\left\{h_{0}+a h^{+}+b h^{-}, a h^{+}+b h^{-} ; 0 \leqq a, b \leqq 1\right\} .
$$

On the other hand it holds that

$$
\mathscr{I}_{e x}=\left\{0, h_{0}, h^{+}, h^{-}, h_{0}+h^{+}, h_{0}+h^{-}, h^{+}+h^{-}, 1\right\} .
$$

Thus, for this example we see $\mathscr{H}_{e x} \varsubsetneqq \mathscr{H}^{*}$.

\section{Department OF MATHEMATICS, NARA WOMEN'S UNIVERSITY}

\section{References}

[1] W.H. Fleming \& C. Su, Some one dimensional migration models in population genetics theory, Theor. Popul. Biol., 5, 431-449, (1974).

[2] R. Holley \& T. Liggett, Ergodic theorems for weakly interacting infinite systems and the voter model, Ann. Prob., 3, 643-663, (1975).

[3] M. Kimura \& G. Weiss, The stepping stone model of population structure and decrease of genetical correlation with distance, Genetics, 49, 561-576, (1964).

[4] T. Liggett, A characterization of the invariant measures for an infinite particle system with interactions, I and II, Trans. Amer. Math. Soc., 179, 433-453, (1973), 198, 201-213, (1974).

[5] T. Liggett, The stochastic evolutions of infinite systems of interacting particles, Lecture Note in Mathematics 598, 187-248, Springer-Verlag, (1977).

[6] N. Matloff, Ergodicity conditions for dissonant voting model, Ann. Prob. 5, 371384, (1977).

[7] T. Maruyama, Stochastic problems in population genetics, Lecture Note in Biomathematics, 17, Springer-Verlag, (1977).

[8] T. Nagylaki, The decay of genetic variability in geographically structured populations, Proc. Nat. Acad. Sci. USA, 71, 2932-2936, (1976).

[9] S. Sawyer, Results for the stepping stone model for migration in population genetics, Ann. Prob. 4, 699-728, (1976).

[10] T. Shiga, An interacting system in population genetics, J. Math. Kyoto Univ. 20-2, 213-242, (1980).

[11] T. Shiga \& A. Shimizu, Infinite dimensional stochastic differential equations and their applications. J. Math. Kyoto Univ. 20-3, 395-416, (1980). 